\title{
Linear Interpolation in Pilot Symbol Assisted Channel Estimation for OFDM
}

\author{
Xiaodai Dong, Member, IEEE \\ Department of Electrical and Computer Engineering, \\ University of Victoria, Victoria, British Columbia, Canada V8W 3P6 \\ Email: xdong@ece.uvic.ca \\ Tel: 250 721-6029 Fax: 250 721-6052 \\ Wu-Sheng Lu, Fellow, IEEE \\ Department of Electrical and Computer Engineering, \\ University of Victoria, Victoria, British Columbia, Canada V8W 3P6 \\ Email:wslu@ece.uvic.ca
}

Anthony C.K. Soong, Senior Member, IEEE

Ericsson Wireless Communications, Inc.

Boulder, Colorado, USA 80301

Email: anthony.soong@ericsson.com 


\begin{abstract}
In this paper, we investigate several efficient interpolation techniques for pilot symbol assisted channel estimation in OFDM. The interpolation methods studied include two dimensional (2-D) separable lowpass sinc interpolator with Kaiser window, 2-D separable Deslauriers-Dubuc (DD) interpolation and 2-D discrete Fourier transform (DFT) based lowpass interpolation. The performances of these interpolators are compared with those of the well known minimum mean-square error (MMSE) 2-D separable Wiener filter and the perfect channel state information. It is shown that the Kaiser window based interpolator and DD interpolation are simple, robust, and outperform the 2-D DFT based lowpass interpolation as well as several existing interpolation methods proposed in the literature. These two schemes are suitable candidates for use in 1-D and 2-D channel estimation.
\end{abstract}

\title{
Index Terms
}

OFDM, pilot symbol assisted channel estimation, Deslauriers-Dubuc (DD) interpolation, sinc interpolator, Kaiser window, two-dimensional (2-D) interpolation

\section{INTRODUCTION}

Orthogonal Frequency Division Multiplexing (OFDM) is a very promising technology for the long-term evolution of $3 \mathrm{G}$ wireless cellular systems [1] and is currently used in several broadcast systems (e.g., [2]- [3]). Moreover, it is well known that the performance of an OFDM system can be greatly influenced by the accuracy of the channel estimation. Consequently, there is, currently, in the cellular communication industry great interest in channel estimation techniques that are compatible with OFDM systems. One promising technique is pilot symbol assisted channel estimation, because of its simple implementation and accurate and robust performance. This scheme inserts known pilot symbols periodically both in the time and frequency dimension to track the time variation and frequency selectivity of the channel. At the receiver, the channel complex gain at the pilot symbol positions can be easily obtained from the received signal and the known pilot symbols. Interpolation is then applied to derive the estimation of the channel knowledge at data symbol positions. Pilot patterns and interpolation methods have been 
extensively studied in the literature. Two types of pilot patterns, namely, block pilots [4] and comb pilots, have been widely used [5] (and the references therein), while other two-dimensional (2-D) pilot patterns were discussed in [6], [7]. Comb pilots based channel estimation and time and frequency domain scattered pilots usually perform better than block type pilot pattern in fast fading channels in tracking the time variation of the channel. Five different pilot patterns were compared in [8], one of which was a novel channel statistics dependent design that leads to reduced bit error rate or the number of pilot symbols required.

The minimum mean-square error (MMSE) channel estimation for block pilots was studied in [4]. The 2-D optimum wiener filtering for pilot symbol assisted OFDM channel estimation was first derived in [9]. Since the optimum Wiener filter requires the channel statistics, a robust interpolator was proposed in [7], which is a diamond shape lowpass filter implemented in the transform domain by 2-D fast Fourier transform (FFT) and inverse FFT (IFFT). A diamond pilot pattern was used in [7]. It was first proposed in [10] to use discrete Fourier transform (DFT), zero-padding and inverse DFT (IDFT) for lowpass interpolation in the transform domain. Linear interpolation, second-order polynomial interpolation, spline, transform domain interpolation and lowpass interpolation using MATLAB built-in function interp for comb pilots were investigated in [5]. A novel coded decision directed demodulation of coherent OFDM was proposed in [11] and an orthogonal coded pilot OFDM with maximum likelihood sequence estimation was investigated in [12]. Recently, optimum pilot patterns for linear interpolators were derived analytically in [13], and performance analysis and design of the linear interpolator followed by a 2-D moving average finite impulse response (MA FIR) filter with rectangular pilot grid was presented in [14]. Separating smoothing and interpolation in the channel estimation was another approach addressed in [15] and [16], where comb type pilot sub-carrier arrangement was adopted. A low rank minimum mean-square error algorithm was employed for smoothing, followed by linear and second order polynomial interpolation used in [15] while a mismatched Wiener interpolation filter used in [16]. 
In this paper, three new interpolation techniques for OFDM channel estimation are proposed. These include a Kaiser window based 2-D sinc interpolator, a 2-D Deslauriers-Dubuc (DD) interpolation method, and a 2-D DFT lowpass interpolation method. Interpolation principle of each proposed method is also presented along with its relevant properties and implementation consideration. The rest of the paper is organized as follows. Section II presents the OFDM system model with pilot symbol assisted channel estimation. Section III concentrates on the three new interpolation techniques and the 2-D MMSE interpolation filter. Section IV summarizes simulation results and Section $\mathrm{V}$ presents a comparison study on the performance and complexity of the four interpolation methods. Finally, conclusions are given in Section VI.

\section{SYSTEM MODEL}

In an OFDM system, information data are transmitted in blocks. Denote the $k$-th data in the $n$-th block by $x[n, k]$, where $x[n, k] \in \mathcal{M}$ is from a 2-D $M$-ary signaling set $\mathcal{M}$ with average energy $E_{s}$ in each dimension, $0 \leq k \leq K-1,0 \leq n \leq N-1, K$ is the total number of sub-carriers and $N$ is the total number of data blocks. Denote pilot symbols inserted in both the time and frequency dimension by $x\left[n_{p}, k_{q}\right]$. In this paper, we consider rectangular grid of the pilot symbols since it is widely used in practical implementation. For a rectangular grid, $n_{p}=p d_{t}$ and $k_{q}=q d_{f}$, where $d_{t}$ and $d_{f}$ are the pilot intervals in terms of number of symbols in the time and frequency domain, respectively, $0 \leq p \leq\left\lfloor\frac{N}{d_{t}}\right\rfloor-1,0 \leq q \leq\left\lfloor\frac{K}{d_{f}}\right\rfloor-1$, and $\lfloor\cdot\rfloor$ is the floor operator.

The pilot inserted OFDM symbol block is then passed through an inverse Fast Fourier transform, parallel to serially converted and added with a guard interval in the form of cyclic prefix $(\mathrm{CP})$ before transmitting to a wireless channel. Consider a time varying frequency selective channel $h(t, \tau)$ given by

$$
h(t, \tau)=\sum_{i=1}^{L} \alpha_{i}(t) \delta\left(\tau-\tau_{i}\right)
$$

where $\alpha_{i}(t)$ and $\tau_{i}$ are the time varying fading gain and delay of the $i$-th path, respectively. The path amplitude $\alpha_{i}(t)$ is a wide sense stationary complex random process and is modeled as a zero 
mean complex Gaussian process in this paper. The fading gains at different paths are assumed independent. Parameter $L$ is the total number of resolvable multi-paths of the channel. Assume that the channel fading at each path is constant within one OFDM symbol with block length $T$ but changes from block to block. The autocorrelation function of each path depends on the normalized maximum Doppler frequency $f_{D} T$. For 2-D isotropic scattering, the autocorrelation function of the $i$-th path can be expressed as

$$
r_{i}(\Delta t)=\mathbb{E}\left[\alpha_{i}^{*}(t) \alpha_{i}(t+\Delta t)\right]=\sigma_{i}^{2} J_{0}\left(2 \pi f_{D} \Delta t\right)=\sigma_{i}^{2} r_{t}(\Delta t)
$$

where $\sigma_{i}^{2}$ is the variance of the path amplitude and $J_{0}(\cdot)$ is the zero-th order Bessel function of the first kind. Without loss of generality, we assume $\sum_{i=1}^{L} \sigma_{i}^{2}=1$.

The channel frequency response at time $t$ is given by

$$
H(t, f)=\int_{-\infty}^{\infty} h(t, \tau) e^{-j 2 \pi f \tau} d \tau=\sum_{i=1}^{L} \alpha_{i}(t) e^{-j 2 \pi f \tau_{i}} .
$$

Sampling (3) at $t=n T$ and $f=k \Delta f$ where $\Delta f=\frac{1}{T}$ is the tone spacing, we have the channel frequency response at the $k$-th tone of the $n$-th OFDM block given by

$$
H[n, k]:=H(n T, k \Delta f)=\sum_{i=1}^{L} \alpha_{i}(n T) e^{-j 2 \pi k \Delta f \tau_{i}} .
$$

Therefore, the auto-correlation function of the frequency response at different blocks and tones is given by [7]

$$
r_{H}(m, l):=\mathbb{E}\left\{H[n+m, k+l] H^{*}[n, k]\right\}=r_{t}(m T) r_{f}(l \Delta f)
$$

where $r_{t}(m T)=J_{0}\left(2 \pi m f_{D} T\right)$ is the time correlation as given in (2) and

$$
r_{f}(l \Delta f)=\sum_{i=1}^{L} \sigma_{i}^{2} e^{-j 2 \pi l \Delta f \tau_{i}} .
$$

The received signal at the $k$-th tone of the $n$-th OFDM block after cyclic prefix removal and fast Fourier transform can be expressed as

$$
y[n, k]=H[n, k] x[n, k]+z[n, k]
$$


where $z[n, k]$ is the zero-mean complex additive white Gaussian noise (AWGN) sample at the $k$-th tone of the $n$-th block with variance $N_{0}$ in its real and imaginary part, respectively. In order to estimate $H[n, k]$, we first obtain the channel estimate at pilot positions by

$$
\tilde{H}_{p}[p, q]=\frac{y\left[n_{p}, k_{q}\right]}{x\left[n_{p}, k_{q}\right]}=H\left[n_{p}, k_{q}\right]+\frac{z\left[n_{p}, k_{q}\right]}{x\left[n_{p}, k_{q}\right]} .
$$

Since the time varying frequency-selective fading channel is bandlimited to the maximum Doppler frequency $f_{D} T$ and has a maximum normalized path delay of $\tau_{L} \Delta f$, the pilot symbol insertion rate $1 / d_{t}$ in the time dimension should satisfy $1 / d_{t} \geq 2 f_{D} T$. Similarly, the pilot insertion rate in the frequency domain should follow $1 / d_{f} \geq 2 \tau_{L} \Delta f$. The channel frequency response at non-pilot positions is now estimated by interpolating the channel estimates at neighboring pilot symbol positions and can be generally expressed as

$$
\hat{H}[n, k]=\sum_{p} \sum_{q} w[n, p ; k, q] \tilde{H}_{p}[p, q]
$$

where $w[n, p ; k, q]$ is a weighting function associated with the particular interpolation method used. Once the complex channel gains at the $k$-th tone of the $n$-th block is estimated, the decision variable is obtained as

$$
D[n, k]=y[n, k] / \hat{H}[n, k]
$$

and $D[n, k]$ is decided as one symbol in the $M$-ary signaling set using the minimum Euclidean distance rule.

\section{INTERPOLATION MEthods}

A number of interpolation methods have been proposed in the literature. The optimum 2-D Wiener filtering was derived in [9] and it was indicated that the cascade of two 1-D Wiener filters achieves performance similar to that of the 2-D Wiener filter with negligible degradation and reduced implementation complexity. A robust interpolator that implements lowpass filtering in the transform domain using 2-D FFT and IFFT was presented in [7]. Other interpolation methods, such as linear, quadratic, and spline interpolation [5], linear interpolation plus 2-D 
moving average [14], have also been investigated. In this paper, we propose 2-D lowpass filter using sinc interpolator with Kaiser window, 2-D Deslauriers-Dubuc interpolation and lowpass filter using 2-D DFT and IDFT.

\section{A. Lowpass Sinc Interpolator with Kaiser Window}

The 1-D sinc interpolator with Kaiser window was extensively studied in [17] for pilot symbol assisted modulation in frequency flat fading channels. It was shown in [17] that the improved performance using Kaiser window over fixed window design is due to the flexible window shape offered by the family of Kaiser window. A detailed procedure on how to determine the various parameters associated with Kaiser window was given. This 1-D design can be extended to the 2-D sinc interpolator with Kaiser window in a straightforward manner.

If pilot symbols are inserted at higher than Nyquist rate of the channel in both the time and frequency dimension, an ideal 2-D brick-wall lowpass filter in the transform domain will reconstruct $H[n, k]$ at non-pilot positions perfectly, if ignoring the noise in the received pilot symbols. Such ideal lowpass filter would require a sinc interpolator with infinite length in both time and frequency dimensions. Simply truncating the sinc interpolator to finite length, i.e., applying a 2-D rectangular window, introduces large passband and stopband fluctuation. The sharpness of the transition band is controlled by the interpolator length. Flexible windows such as Kaiser window provide better performance than fixed window. As detailed in [17], the idea of the Kaiser window design is to push the transition region of the lowpass filter into the zero interval of the channel folded power spectrum and lower the passband and stopband fluctuation in order to minimize the interpolation error caused by the non-ideal lowpass filter. Suppose the 2-D interpolation filter $w_{s k}[n, k]$ has finite number of taps determined by the parameters $M_{t}$ and $M_{f}$, i.e., $-M_{t} d_{t} \leq n \leq M_{t} d_{t},-M_{f} d_{f} \leq k \leq M_{f} d_{f}$. For a symbol position $[n, k]$ and given 
pilot insertion rates of $d_{t}$ and $d_{f}$, the channel estimation at symbol $[n, k]$ can be written as

$$
\begin{aligned}
\hat{H}[n, k] & =\sum_{p=I_{t}-M_{t}} \sum_{q=I_{f}-M_{f}}^{I_{t}+M_{t}} w_{s k}\left[n-n_{p}, k-k_{q}\right] \tilde{H}_{p}[p, q] \\
& =\sum_{u=-M_{t} d_{t}}^{I_{f}+M_{f}} \sum_{v=-M_{f} d_{f}}^{M_{t} d_{t}} w_{s k}[u, v] \hat{H}_{p}[n-u, k-v], \\
\hat{H}_{p}[n, k] & = \begin{cases}\tilde{H}_{p}[p, q], & n=p d_{t}, k=q d_{f} \\
0, & \text { otherwise }\end{cases}
\end{aligned}
$$

where $I_{t}=\left\lfloor\frac{n}{d_{t}}\right\rfloor$ and $I_{f}=\left\lfloor\frac{k}{d_{f}}\right\rfloor$, and $\hat{H}_{p}[n, k]$ is formed by the channel estimation at pilot positions and zero padded at non-pilot positions. The 2-D separable sinc interpolator is given by

$$
w_{s k}\left[n-n_{p}, k-k_{q}\right]=g\left[n-p d_{t}, k-q d_{f}\right] \frac{d_{t} d_{f}}{H_{t} H_{f}} \operatorname{sinc}\left(\frac{n-p d_{t}}{H_{t}}\right) \operatorname{sinc}\left(\frac{k-q d_{f}}{H_{f}}\right)
$$

where

$$
\begin{aligned}
& d_{t} \leq H_{t} \leq \frac{1}{2 f_{D} T} \\
& d_{f} \leq H_{f} \leq \frac{1}{2 \Delta f \tau_{L}}
\end{aligned}
$$

is a result of the Nyquist sampling theorem. Parameters $H_{t}$ and $H_{f}$ determine the sinc lowpass filter cut-off frequencies as $f_{c t}=\frac{1}{2 H_{t} T}$ and $f_{c f}=\frac{1}{2 H_{f} \Delta f}$, respectively [17]. Given $d_{t}, d_{f}, M_{t}$, $M_{f}, H_{t}$ and $H_{f}$, the 2-D Kaiser window function $g[n, k]$ is given by

$$
g[n, k]=\frac{I_{0}\left(\beta_{t} \sqrt{1-\left(\frac{n}{\alpha_{t}}\right)^{2}}\right)}{I_{0}\left(\beta_{t}\right)} \cdot \frac{I_{0}\left(\beta_{f} \sqrt{1-\left(\frac{k}{\alpha_{f}}\right)^{2}}\right)}{I_{0}\left(\beta_{f}\right)},-\alpha_{t} \leq n \leq \alpha_{t},-\alpha_{f} \leq k \leq \alpha_{f}
$$

where $\alpha_{t}=M_{t} d_{t}, \alpha_{f}=M_{f} d_{f}$ and the window size is $2 M_{t} d_{t}+1$ by $2 M_{f} d_{f}+1$. The shape factors $\beta_{t}$ and $\beta_{f}$ are functions of the passband/stopband peak fluctuations $A_{t}$ and $A_{f}$ as given by [17, eq. (19)]. Kaiser window with a different shape factor allows the tradeoff among the interpolation order $M_{t}$, the transition bandwidth, and the passband/stopband peak fluctuation $A_{t}$, given the pilot interval $d_{t}$. The same flexibility exists in the frequency dimension interpolation. Here the peak fluctuations $A_{t}$ and $A_{f}$ are chosen to yield the transition band falling into the 
zero interval of the channel folded power spectrum for given filter lengths, in order to minimize the interpolation error. Following the steps in [17, eqs. (17)-(20)], we have

$$
\begin{aligned}
& A_{t}=\frac{2.285 \cdot 2 \pi\left(1-2 f_{D} T H_{t}\right) 2 M_{t} d_{t}}{H_{t}}+8 \\
& A_{f}=\frac{2.285 \cdot 2 \pi\left(1-2 \Delta f \tau_{L} H_{f}\right) 2 M_{f} d_{f}}{H_{f}}+8 .
\end{aligned}
$$

Note that larger $H_{t}$ and $H_{f}$ lead to smaller cutoff frequencies, and as a result, more noise will be filtered out. This mainly improves the performance in the low to medium range of signal-to-noise ratio (SNR). On the other hand, a smaller cutoff frequency means less transition band allowed, and to meet such requirement, higher interpolation order $M_{t}$ and $M_{f}$ would be needed. Otherwise the designed Kaiser window will have larger peak fluctuation $A_{t}$ and $A_{f}$ and hence more interpolation error that leads to error floors at high SNRs. The relationship between parameters $M_{t}$ (or $M_{f}$ ) and $H_{t}$ (or $H_{f}$ ) can be best explained by [17, Fig. 2].

The 2-D separable lowpass sinc interpolator is essentially the cascade of two 1-D sinc interpolators. Its most efficient implementation is to perform the interpolation of the channel estimation at pilot positions in one dimension and then interpolate in the other dimension. The sinc interpolator is a real time algorithm, which means when one additional pilot symbol is received, a rectangle of $d_{t} \times d_{f}$ data symbols is interpolated. In an OFDM receiver where an OFDM symbol block is processed as a whole, the interpolation in the frequency dimension can be operated on the total available pilot symbols in that block.

The mean-squared error (MSE) of the 2-D linear interpolator is derived in the Appendix.

\section{B. Deslauriers-Dubuc Interpolation}

1) One-dimensional DD interpolation: Deslauriers and Dubuc introduced a multi-scale refinement technique for smooth interpolation of a given set of uniformly spaced data in [18], [19]. The algorithm first finds the midpoint value of each interval by fitting a low order $(D=2 L+1)$ local polynomial using $D+1$ closest data $(L+1$ data to the left of the midpoint to be interpolated and $L+1$ data to the right), where $D=3,5,7, \ldots$ is always an odd integer. By repeating this 
algorithm again and again, each time doubling the number of data points or nodes by midpoint interpolation, eventually a dense set of interpolated data points are obtained. As can be seen from this procedure, the number of interpolated data between two originally given data is $2^{J}-1$, where $J$ denotes the interpolation density. Since the interpolation of one midpoint involves only $D+1$ closest data from the previous iteration, DD interpolation is a local algorithm that leads to smooth results.

Denote a given data set $\left\{f\left(k \Delta_{x}\right)\right\}$ by $\left\{f_{k}\right\}$, where $\Delta_{x}$ is the uniform distance between data, $k \in \mathcal{Z}$. Without loss of generality, we treat the input data set $\left\{f_{k}\right\}$ as defined at integers. Suppose we want to interpolate $2^{J}$ points for each interval, i.e., to insert $2^{J}-1$ points between two successive data. The interpolated data are at positions $k+n / 2^{J}, n=1, \ldots, 2^{J}-1$. The aforementioned iterative process can be implemented in a simple form by the interpolating function as [20]

$$
\tilde{f}(x)=\sum_{k \in \mathcal{Z}} f_{k} \phi_{D}(x-k)
$$

where $\phi_{D}(x)$ is called the fundamental function or interpolating function of the DD interpolation scheme. Eq. (16) is simply a linear combination of the weighted and shifted fundamental function.

In DD interpolation, the fundamental function $\phi_{D}(x)$ is defined iteratively at any rational number whose denominator is an integer power of base $b$. Here the subscript $D$ indicates the degree of the local polynomial used in the iteration process. For practical purposes, the most useful base is $b=2$ [20], which is used in our problem formulation. In this case, the generation of the $D$-th order local polynomial based fundamental function $\phi_{D}(x)$ with density $J$ starts with the Kronecker sequence $\left\{\phi_{D}(k), k \in \mathcal{Z}\right\}$, which assumes $\phi_{D}(0)=1$ and $\phi_{D}(k)=0$ for all nonzero integer $k$. The DD refinement then proceeds iteratively as follows: Let $j=0, D$ be an odd positive integer, and $L$ be the integer such that $D=2 L+1$. Given the values of $\phi_{D}(x)$ at $\left\{(k-L) / 2^{j}, \ldots,(k+L+1) / 2^{j}\right\}$, the values of $\phi_{D}(x)$ at $(k+0.5) / 2^{j}$ is determined by fitting a $D$-th order polynomial to the above data set. The iteration is supposed to be carried out for all integers $k$. However, since the iteration starts with the Kronecker sequence, one needs to do 
no more than $L+1$ polynomial fittings. In the next iteration with $j=1$, the above steps are repeated for all integers $k$, but again only a limited number of polynomial fittings are needed because only finite number of nonzero $\phi_{D}\left(k / 2^{j}\right)$ exist. The iteration continues until $j$ reaches the given value $J$. Example plots of the fundamental function $\phi_{D}(x)$ with different order $D$ and interpolation density $J$ are given in Fig. 1 .

The DD interpolation process defines a unique, uniformly continuous function that actually belongs to function class $\mathcal{C}^{\alpha}$ with $\alpha=\alpha(D)$ a monotonically increasing function of $D$ [18], [19]. An important property of the fundamental function $\phi_{D}(x)$ is that its region of support is finite and is within the interval $[-D, D]$. This property becomes transparent when one realizes that $\phi_{D}(x)$ may be regarded as the autocorrelation function of the orthogonal Daubechies scaling function $s_{D}(x)$ of length $D+1$ [23]. Since $s_{D}(x)$ is known to have a region support on $[0, D]$, its autocorrelation function has a region of support on $[-D, D]$. From this property it follows that the value of $\tilde{f}(x)$ at a given point $x$ depends only on the data samples $\left\{f_{k}\right\}$ that are near by the point $x$, and it is the value of $D$ that controls how local this dependence will be.

2) Implementation of $D D$ interpolation: Let $\phi_{D}(x)$ in (16) be the fundamental function generated by the DD algorithm using $D$-th order local polynomial with density $J$. Denote the $2^{J}-1$ interpolated samples on interval $(i-1, i)$ by $\mathbf{f}_{(i-1, i)}^{(J)}$ and the $2^{J}-1$ samples of $\phi_{D}(x)$ on interval $(k-1, k)$ by $\phi_{D, k}^{(J)}$. It follows from (16) and the finite region of support property that the entire block of interpolated data $\mathbf{f}_{(i-1, i)}^{(J)}$ can be generated as a weighted sum of 2D blocks of known data $\left\{\phi_{D, k}^{(J)},-D+1 \leq k \leq D\right\}$ with the known signal samples $\left\{f_{i-k},-D+1 \leq k \leq D\right\}$ as the weights:

$$
\mathbf{f}_{(i-1, i)}^{(J)}=\sum_{k=-D+1}^{D} f_{i-k} \boldsymbol{\phi}_{D, k}^{(J)} .
$$

Fig. 2 illustrates the formula in (17) with $i=3$ and $D=3$. If we view each $\phi_{D, k}^{(J)}$ in (17) as a column vector with $2^{J}-1$ components, and $\mathbf{f}_{(i-1, i)}^{(J)}$ as a column vector of the same dimension, then (17) can be expressed as

$$
\mathbf{f}_{(i-1, i)}^{(J)}=\boldsymbol{\Phi}_{D}^{(J)} \mathbf{f}_{D, i}
$$


where matrix $\boldsymbol{\Phi}_{D}^{(J)}=\left[\boldsymbol{\phi}_{D,-D+1}^{(J)}, \boldsymbol{\phi}_{D,-D+2}^{(J)}, \cdots, \boldsymbol{\phi}_{D, D}^{(J)}\right]$ and $\mathbf{f}_{D, i}=\left[f_{i+D-1}, f_{i+D-2}, \cdots, f_{i-D}\right]^{T}$. Note that matrix $\boldsymbol{\Phi}_{D}^{(J)}$ can be readily constructed from $\phi_{D}(x)$ by dividing it over its region of support $[-D, D]$ into $2 D$ equal parts (excluding its values at integer points) and put them together as $2 D$ column vectors. For a given degree $D$ and density $J$, this data array can be pre-calculated and stored, and formula (18) in this case serves as the basis for an efficient implementation scheme that produces interpolated samples block by block as the incoming data sample updates vector $\mathbf{f}_{D, i}$ with index $i$ representing the current time or frequency.

Alternatively, the interpolation can be achieved by inserting $2^{J}-1$ zeros between every two successive data samples and then convolving the zero-inserted data sequence with $\phi_{D}(x)$ of density $J$.

3) 2-D DD interpolation: The 2-D counterpart of (16) is given by

$$
\tilde{f}(t, f)=\sum_{k, l \in \mathcal{Z}^{2}} f_{k, l} \phi_{2}(t-k, f-l)
$$

where $\left\{f_{k, l},(k, l) \in \mathcal{Z}^{2}\right\}$ is a 2-D array of data samples and $\phi_{2}(t, f)$ is a 2-D fundamental function satisfying

$$
\phi_{2}(t, f)= \begin{cases}1 & \text { for }(t, f)=(0,0) \\ 0 & \text { for }(t, f)=\text { integer pair not equal to }(0,0) .\end{cases}
$$

For the sake of implementation efficiency, we consider the separable $\phi_{2}(t, f)$ which assumes the form

$$
\phi_{2}(t, f)=\phi_{D_{t}}^{\left(J_{t}\right)}(t) \phi_{D_{f}}^{\left(J_{f}\right)}(f)
$$

where $\phi_{D_{t}}^{\left(J_{t}\right)}(t)$ and $\phi_{D_{f}}^{\left(J_{f}\right)}(f)$ are 1-D fundamental functions obtained by the DD algorithm with $D_{t^{-}}$ th and $D_{f}$-th local polynomials with density $J_{t}$ and $J_{f}$, respectively. Note that (21) in conjunction with the finite region of support for the 1-D DD fundamental function implies that $\phi_{2}(t, f)$ in (21) has the finite region of support $\left[-D_{t}, D_{t}\right] \times\left[-D_{f}, D_{f}\right]$. Applying the 2-D DD interpolation to the 2-D OFDM channel estimation problem, the channel's transfer function at the $[n, k]$ position 
is estimated as

$$
\hat{H}[n, k]=\sum_{q=I_{f}-D_{f}+1}^{I_{f}+D_{f}} \sum_{p=I_{t}-D_{t}+1}^{I_{t}+D_{t}} \tilde{H}_{p}[p, q] \phi_{D_{t}}\left(n / d_{t}-p\right) \phi_{D_{f}}\left(k / d_{f}-q\right)
$$

where for notation simplicity $\phi_{D_{t}}(t)$ and $\phi_{D_{f}}(f)$ denote $\phi_{D_{t}}^{\left(J_{t}\right)}(t)$ and $\phi_{D_{f}}^{\left(J_{f}\right)}(f)$, respectively, and $D_{t}$ and $D_{f}$ are the order of the DD interpolation in the time and frequency dimension, respectively. Note that DD interpolation requires $d_{t}=2^{J_{t}}$ and $d_{f}=2^{J_{f}}$, where $J_{t} \in \mathcal{Z}, J_{f} \in \mathcal{Z}$.

We remark that when the orders $D_{t}$ and $D_{f}$ and/or density $J_{t}$ and $J_{f}$ are different, the two implementations of (22), i.e.,

$$
\hat{H}[n, k]=\sum_{q=I_{f}-D_{f}+1}^{I_{f}+D_{f}}\left(\sum_{p=I_{t}-D_{t}+1}^{I_{t}+D_{t}} \tilde{H}_{p}[p, q] \phi_{D_{t}}\left(n / d_{t}-p\right)\right) \phi_{D_{f}}\left(k / d_{f}-q\right)
$$

and

$$
\hat{H}[n, k]=\sum_{p=I_{t}-D_{t}+1}^{I_{t}+D_{t}}\left(\sum_{q=I_{f}-D_{f}+1}^{I_{f}+D_{f}} \tilde{H}_{p}[p, q] \phi_{D_{f}}\left(k / d_{f}-q\right)\right) \phi_{D_{t}}\left(n / d_{t}-p\right)
$$

will have different complexity. The number of multiplications for producing one interpolating sample in these two implementations are found to be approximately equal to $2 D_{t}\left(1+4 D_{f} / d_{f}\right)$ and $2 D_{f}\left(1+4 D_{t} / d_{t}\right)$, respectively, based on which an efficient implementation scheme can be chosen.

For true 2-D DD interpolations, the fundamental function $\phi_{2}(t, f)$ cannot be separated as the product of two 1-D fundamental functions. Our derived 2-D non-separable fundamental function is found to have negligible difference relative to the 2-D separable fundamental function and the resulting interpolation and error performance are almost identical to that of the separable 2-D interpolation. However, 2-D non-separable DD has much higher complexity. Therefore, 2-D separable DD interpolation implemented as the cascade of two 1-D interpolation should be used in practical systems.

\section{2-D DFT Based Lowpass Interpolation}

Application of 1-D DFT based lowpass interpolation to the comb pilot case was first investigated in [10]. This technique involves the insertion of a fixed number of zeros into the centre 
of the DFT of the data sequence, followed by inverse DFT of the modified DFT sequence and scaling. It is essentially a process of zero-padding plus ideal lowpass filtering, and see Sec. 10.3 of [21] for detailed elaboration of this technique.

In an effort to extend the technique to the 2-D case, it is realized that the zero insertion should be such that pushes the data away from the centre in both dimensions. The proposed extension can be described as follows. Suppose there are $N_{p} \times K_{q}$ pilot channel estimates $\tilde{\mathbf{H}}_{p}$. Denote the 2-D DFT of $\tilde{\mathbf{H}}_{p}$ by $\mathbf{V}$. Now cut $\mathbf{V}$ into four blocks: $\mathbf{V}_{11}=\mathbf{V}\left(1: \frac{N_{p}+1}{2}, 1: \frac{K_{q}+1}{2}\right)$, $\mathbf{V}_{12}=\mathbf{V}\left(1: \frac{N_{p}+1}{2}, \frac{K_{q}+1}{2}+1: K_{q}\right), \mathbf{V}_{21}=\mathbf{V}\left(\frac{N_{p}+1}{2}+1: N_{p}, 1: \frac{K_{q}+1}{2}\right)$ and $\mathbf{V}_{22}=\mathbf{V}\left(\frac{N_{p}+1}{2}+1:\right.$ $N_{p}, \frac{K_{q}+1}{2}+1: K_{q}$ ), and put these four blocks at the four corners of a $d_{t} N_{p} \times d_{f} K_{q}$ matrix $\mathbf{V}_{y}$ where the rest of the entries are all zero. Finally, take the inverse DFT of $\mathbf{V}_{y}$ to obtain the interpolated data. The above assumes $N_{p}$ and $K_{q}$ are odd numbers. If this is not the case, slight modifications as in the 1-D case detailed in [21] should be adopted. The cut-off frequencies of this 2-D interpolation are $f_{c t}=\frac{1}{2 d_{t} T}$ and $f_{c f}=\frac{1}{2 d_{f} \Delta f}$, respectively.

\section{Minimum Mean-Square Error Interpolation}

The optimum MMSE interpolation has been well studied in the literature [9], [7]. It was concluded in [9] that two 1-D Wiener filters, i.e., 2-D separable Wiener filter, achieves negligible performance degradation to the optimum 2-D non-separable Wiener filter, with much reduced complexity. The 1-D Wiener filter for interpolating the $k$-th sub-carrier channel from its neighboring pilot sub-carriers in the $n_{p}$-th OFDM block is given by

$$
\mathbf{w}_{f}[k]=\left(\mathbf{R}_{H H}^{f}+\frac{N_{0}}{E_{s}} \mathbf{I}\right)^{-1} \mathbf{r}_{H k}^{f}
$$

where $\mathbf{I}$ is the identity matrix, $\mathbf{R}_{H H}^{f}=\mathbb{E}\left[\mathbf{H}_{p k} \mathbf{H}_{p k}^{H}\right]$ is the auto-correlation matrix of channel fadings at pilot sub-carriers with window length $M_{s}^{f}$, and the superscript $H$ indicates Hermitian transpose. The vector $\mathbf{r}_{H k}^{f}=\mathbb{E}\left[\mathbf{H}_{p k} H^{*}\left[n_{p}, k\right]\right]$ is the cross-correlation between the current symbol and the channel fadings at pilot sub-carriers. The pilot symbol channel vector $\mathbf{H}_{p k}$ is

$$
\mathbf{H}_{p k}=\left[H\left[n_{p},\left(I_{f}-M_{1}^{f}\right) d_{f}\right], H\left[n_{p},\left(I_{f}-M_{1}^{f}+1\right) d_{f}\right], \ldots, H\left[n_{p},\left(I_{f}+M_{2}^{f}\right) d_{f}\right]\right]^{T}
$$


where $I_{f}=\left\lfloor\frac{k}{d_{f}}\right\rfloor, M_{1}^{f}=\left\lfloor\frac{M_{s}^{f}-1}{2}\right\rfloor$ and $M_{2}^{f}=\left\lfloor\frac{M_{s}^{f}}{2}\right\rfloor$. From (5) and (6), the $(u, v)$-th element of $\mathbf{R}_{H H}^{f}$ is given by

$$
R_{H H}^{f}[u, v]=\sum_{i=1}^{L} \sigma_{i}^{2} e^{-j 2 \pi(u-v) d_{f} \Delta f \tau_{i}}, \quad 0 \leq u \leq M_{s}^{f}-1,0 \leq v \leq M_{s}^{f}-1
$$

which is independent of $n_{p}$ and $k$, and

$$
r_{H k}^{f}[u]=\sum_{i=1}^{L} \sigma_{i}^{2} e^{-j 2 \pi\left(\left(I_{f}-M_{1}^{f}+u\right) d_{f}-k\right) \Delta f \tau_{i}}, \quad 0 \leq u \leq M_{s}^{f}-1 .
$$

The channel estimate at $\left[n_{p}, k\right]$ is then given by

$$
\tilde{H}\left[n_{p}, k\right]=\mathbf{w}_{f}^{H}[k] \tilde{\mathbf{H}}_{p k}
$$

where $\tilde{\mathbf{H}}_{p k}=\left[\tilde{H}_{p}\left[n_{p},\left(I_{f}-M_{1}^{f}\right) d_{f}\right], \tilde{H}_{p}\left[n_{p},\left(I_{f}-M_{1}^{f}+1\right) d_{f}\right], \ldots, \tilde{H}_{p}\left[n_{p},\left(I_{f}+M_{2}^{f}\right) d_{f}\right]\right]^{T}$. Similarly, the 1-D Wiener filter for interpolation at the time dimension is given by

$$
\mathbf{w}_{t}[n]=\left(\mathbf{R}_{H H}^{t}+\frac{N_{0}}{E_{s}} \mathbf{I}\right)^{-1} \mathbf{r}_{H n}^{t}
$$

where $\mathbf{R}_{H H}^{t}=\mathbb{E}\left[\mathbf{H}_{n k} \mathbf{H}_{n k}^{H}\right], \mathbf{r}_{H n}^{f}=\mathbb{E}\left[\mathbf{H}_{n k} H^{*}[n, k]\right]$, and

$$
\mathbf{H}_{n k}=\left[H\left[\left(I_{t}-M_{1}^{t}\right) d_{t}, k\right], H\left[\left(I_{t}-M_{1}^{t}+1\right) d_{t}, k\right], \ldots, H\left[\left(I_{t}+M_{2}^{t}\right) d_{t}, k\right]\right]^{T} .
$$

Moreover, $I_{t}=\left\lfloor\frac{n}{d_{t}}\right\rfloor, M_{1}^{t}=\left\lfloor\frac{M_{s}^{t}-1}{2}\right\rfloor$ and $M_{2}^{t}=\left\lfloor\frac{M_{s}^{t}}{2}\right\rfloor$. From (5), we have

$$
R_{H H}^{t}[u, v]=J_{0}\left(2 \pi|u-v| d_{t} f_{D} T\right), \quad 0 \leq u \leq M_{s}^{t}-1,0 \leq v \leq M_{s}^{t}-1
$$

and

$$
r_{H n}^{t}[u]=J_{0}\left(2 \pi\left|\left(I_{t}-M_{1}^{t}+u\right) d_{t}-n\right| f_{D} T\right), \quad 0 \leq u \leq M_{s}^{t}-1 .
$$

Finally, the channel estimate at $[n, k]$ is obtained as

$$
\hat{H}[n, k]=\mathbf{w}_{t}^{H}[n] \tilde{\mathbf{H}}_{n k}
$$

where $\tilde{\mathbf{H}}_{n k}=\left[\tilde{H}\left[\left(I_{t}-M_{1}^{t}\right) d_{t}, k\right], \tilde{H}\left[\left(I_{t}-M_{1}^{t}+1\right) d_{t}, k\right], \ldots, \tilde{H}\left[\left(I_{t}+M_{2}^{t}\right) d_{t}, k\right]\right]^{T}$. 


\section{Performance Evaluation}

In this section, the four types of interpolation techniques discussed in Section III are simulated for an OFDM system setup summarized in Table I.The effect of different parameters of each interpolation method on the performance are investigated in this section.

Before we proceed to the simulation results for channel estimation of OFDM systems, the performance of pilot symbol assisted 64-QAM in frequency flat Rayleigh fading with $f_{D} T=0.03$ is first presented for sinc interpolator with Kaiser window and DD interpolation in Figs. 3 and 4. Note that average energy per bit $E_{b}=E_{s} / 6$ for 64-QAM. The SNR definition in all figures is the average energy per bit for both pilot and data symbols, not the effective SNR for data bits only. It can be seen from Fig. 3 that for $d_{t}=4$ and $d_{t}=8$, DD interpolation achieves the same performance as a Kaiser window based sinc interpolator with properly chosen parameters. The number of pilot symbols involved in the DD interpolator $D$ is equal to that of the Kaiser window interpolation if pilot symbols are inserted frequently, i.e., $d_{t}=4$. When $d_{t}=8$, the order of the DD interpolation should be $D=9$ and about twice the Kaiser window interpolation order $M_{t}=4$. As explained in Section III-A, increasing Kaiser window parameter $H_{t}$ while satisfying (13) effectively reduces the bandwidth of the lowpass filter and thus has denoising effect, but leads to higher interpolation error that may be partially compensated by a larger $M_{t}$. Fig. 3 shows that for the same $d_{t}=4$, a larger value of $H_{t}=11$ leads to power savings at the low to medium range of SNR but higher error floors as SNR increases. It is found in our study that higher order $D$ in DD interpolation or $M_{t}$ in the sinc interpolator is required when the pilot symbols are spaced far apart, or the channel is rapidly varying.

Fig. 4 depicts the bit error rate (BER) of pilot symbol assisted modulation (PSAM) 64-QAM as a function of interpolation order $M_{t}$. For a given SNR and $H_{t}$, a larger $M_{t}$ yields lower BER. However, increasing $M_{t}$ beyond certain values no longer leads to BER reduction. This is because passband and stopband fluctuation can not be completely removed with the increase of $M_{t}$. Since $M_{t}$ determines the filter length and hence implementation complexity, $M_{t}$ should not 
be chosen larger than necessary.

The channels used for OFDM simulation are ITU Vehicular A channels [24] and COST 259 typical urban channels [25] with mobile speed at $30 \mathrm{~km} / \mathrm{h}$ and $120 \mathrm{~km} / \mathrm{h}$. Fig. 5 depicts the bit error rate of the OFDM system detailed in Table I using five different interpolators. Fig. 5 shows that both DD and the sinc interpolator with Kaiser window can achieve almost the same performance as interp and no error floor for the range of SNR simulated. It was shown in [5] that interp produced the best performance for 1-D comb pilot based channel estimation among the five interpolation methods studied in that paper. There is less than $1 \mathrm{~dB}$ degradation using DD/Kaiser interpolation relative to perfect channel state information. 2-D DFT lowpass interpolation is the fastest algorithm but achieves relatively poor performance, especially at high SNRs with a severe error floor. As expected, MMSE has the best performance that is close to the perfect channel estimation. The MSEs of the interpolators studied are shown in Fig. 6, indicating larger performance difference among interpolators.

Fig. 7 shows the effect of various parameters of Kaiser window on the performance. Choosing $H_{t}=d_{t}=4$ and $H_{f}=d_{f}=4$ results in error floor free performance for the SNR range studied. The interpolation order $M_{t}=M_{f}=2$ yields slightly lower BER than $M_{t}=M_{f}=10$. Therefore, $M_{t}$ and $M_{f}$ should not be selected larger than necessary. Furthermore, increasing $H_{t}$ and $H_{f}$ slightly closes the gap to the ideal channel estimation at low to medium SNR values $(\leq 20 \mathrm{~dB})$ due to its de-noising capability. However, they lead to larger interpolation error and thus higher error floor at larger SNR values.

Fig. 8 demonstrates the effect of different parameters in DFT based, DD and MMSE interpolation on the BER performance in COST 259 typical urban channels with large maximum Doppler frequency. DFT lowpass filter is sensitive to the block size of the available data for interpolation. Reducing $\left(N_{p}, K_{q}\right)=(128,64)$ pilot blocks to $(80,64)$ degrades the performance. Large block size, however, requires more storage space and results in larger latency. For DD interpolation, using a higher order $D_{t}=D_{f}=7$ actually slightly increases the BER compared to 
$D_{t}=D_{f}=3$. Therefore, $D_{t}=D_{f}=3$ is sufficient for the pilot spacing, the channel fading rate and the channel frequency selectivity studied. For the MMSE channel estimation, the interpolation coefficients (25) and (30) optimized for each operating SNR has the best performance. Since this is practically impossible, choosing a fixed SNR operating point at $E_{b} / N_{0}=40 \mathrm{~dB}$ suffers little degradation.

Figs. 9 and 10 depict the BER and MSE of the OFDM system with pilot spacing $d_{t}=d_{f}=8$ in a low mobility typical urban channel respectively. Again, DD interpolation performs equally well as the Kaiser window based sinc interpolator with suitably chosen parameters. The interp channel estimator in terms of MSE clearly outperforms the DD and Kaiser interpolators, but the resulting BERs have less gap. The filter length of the DD and Kaiser interpolators are longer compared to Fig. 8 as pilot symbols are spaced further apart.

\section{COMPLEXITY And Discussion}

The complexity of each interpolator is tabularized in Table II by means of the number of complex multiplication operations required. For 2-D separable interpolators, two complexity numbers are given depending interpolation is performed on which dimension first. Block processing in the table refers to the scenario where a whole block of received OFDM symbols is read in each time for interpolation. Discussion on the properties and suitability of the four interpolators is presented below.

Lowpass sinc interpolator with Kaiser window and DD interpolation. Properly designed Kaiser window based lowpass sinc interpolator achieves similar performance to DD interpolation. Both schemes are robust since they do not require channel statistics and are system operating point independent. In a practical scenario, the useful channel information that guides the determination of interpolator parameters is the maximum possible Doppler frequency, the maximum possible multipath delay and the operating SNR range. Note that they are not the exact operating parameters of the particular channel. If pilot symbol insertion rate is much higher than the Nyquist rate of the channel in either dimension, $M_{t}$ and/or $M_{f}$ of Kaiser window and $D_{t}$ 
and/or $D_{f}$ of DD interpolator can be rather small. In this case, Kaiser window provides an additional parameter $H_{t}$ and/or $H_{f}$ with denoising capability to improve the BER performance in the low to medium range of SNR values, together with larger $M_{t}$ and/or $M_{f}$. If pilot insertion rate is close to the Nyquist rate, the interpolation order of the Kaiser window and DD interpolator need to be relatively large, and the $H_{t}$ and/or $H_{f}$ parameters of Kaiser window should be set the same as $d_{t}$ and $d_{f}$. Finally, if the operating SNR stretches to a large range, larger interpolation order will help to lower the error floor at high SNRs. In practice, the interpolation order is often limited by the hardware resources of the receiver.

In many practical cases, low orders of both interpolators are sufficient, which translates into low complexity and small latency. DD interpolation is simpler than Kaiser window, with less parameters to determine. Pre-computation of fundamental functions is also more easily implementable than Kaiser window because once the density $J$ is fixed, only a small number of fundamental functions with different order $D$ needs to be calculated. On the other hand, Kaiser window offers more flexibility in performance tuning and pilot spacing. It is also found that in some cases larger interpolator order is required for DD to achieve a similar performance to Kaiser window. Hybrid interpolation methods such as using DD for one dimension and Kaiser window based sinc interpolator for the other dimension may be employed if pilot spacing in one dimension is not a power of 2. Furthermore, DD interpolation has no de-noising effect when there is noise in the given data, despite of its powerful capability in performing interpolation. One possible solution is to combine DD with de-noising/smoothing algorithms. For example, the low rank MMSE noise reduction algorithm in [16] can be employed and followed by DD interpolation.

2-D DFT based lowpass interpolation. Performing lowpass interpolation through 2-D DFT/IDFT is the most efficient to implement, if the DD and Kaiser window interpolators do not precompute, respectively, the fundamental function and the window function. The 2-D DFT lowpass interpolation is also robust and does not require channel statistics. The drawback of this method 
is the less desirable performance. Moreover, since a large number of observed data is needed to obtain good interpolation performance, 2-D DFT method is not real time, incurs large latency and requires large data storage.

MMSE interpolation. MMSE interpolation provides optimum performance however with requirement of knowledgement of channel statistics (i.e., channel autocorrelation function) in both time and frequency dimension and the signal-to-noise ratio that the system is operating at. Such information may not be available in practice. Furthermore, two 1-D Wiener filters involve $M_{s}^{t} \times M_{s}^{t}$ and $M_{s}^{f} \times M_{s}^{f}$ matrix inverse that has high complexity $\mathcal{O}\left(\left(M_{s}^{t}\right)^{3} / 6\right)$ and $\mathcal{O}\left(\left(M_{s}^{f}\right)^{3} / 6\right)$.

\section{CONCLUSION}

DD interpolation and the sinc interpolator with Kaiser window have been proposed and studied in this paper. It has been shown that both interpolators are robust, system operating point independent and do not require channel statistics. They are simpler to implement than Wiener filters that require matrix inverse operation and channel statistics, and provide better performance than 2-D DFT lowpass interpolation. DD interpolator has even lower complexity than Kaiser window based sinc interpolator, while the latter offers more flexibility in cases where DD is not applicable. These two channel estimation techniques are promising for use in future wireless OFDM communication systems.

\section{REFERENCES}

[1] Ericsson, Fujitsu, Motorola, Nokia, NTT DoCoMo, Panasonic and Siemens, "Basic principles for the evolved UTRA radio-access concept,”3GPP TSG-RAN WG1 R1-050448, May 2005.

[2] ETSI EN 300744 v1.5.1, "Digital Video Broadcasting (DVB); Framing structure, channel coding and modulation for digital terrestrial television," Nov. 2004.

[3] 3GPP2 C.S0054 v1.2, "cdma2000 High Rate Broadcast-Multicast Packet Data Air Interface Specification (draft text)," 3GPP2-C30-20050718-016, July 2005.

[4] J.-J. van de Beek, O. Edfors, M. Sandell, S. K. Wilson and P. O. Börjesson, "On channel estimation in OFDM systems," Proc. IEEE Vehicular Technology Conference (VTC'95), vol. 2, Chicago, IL, pp. 815-819, July 1995. 
[5] S. Coleri, M. Ergen, A. Puri and A. Bahai, "Channel estimation techniques based on pilot arrangement in OFDM systems," IEEE Trans. Broadcasting, pp. 223-229, Sept. 2002.

[6] M. J. Fernández-Getino García, S. Zazo and J. M. Páez-Borrallo, "Pilot patterns for channel estimation in OFDM," Electronics Letters, vol. 36, no. 12, pp. 1049-1050, June 2000.

[7] Y. Li, "Pilot-symbol-aided channel estimation for OFDM in wireless systems", IEEE Trans. Vehicular Techno., pp. 12071215, July 2000.

[8] F. Tufvesson and T. Maseng, "Pilot assisted channel estimation for OFDM in mobile cellular systems," Proc. IEEE VTC'97, pp. 1639-1643, May 1997.

[9] P. Hoeher, S. Kaiser and P. Robertson, "Two-dimensional pilot-symbol-aided channel estimation by Wiener filtering," IEEE Intl. Conf. Acoustics, Speech, and Signal Processing (ICASSP'97), Munich, Germany, pp. 1845-1848, Apr. 1997.

[10] Y. Zhao and A. Huang, "A novel channel estimation method for OFDM mobile communication systems based on pilot signals and transform-domain processing," IEEE 47th Vehicular Techno. Conf. (VTC'97), pp. 2089-2093, May 1997.

[11] V. Mignone and A. Morello, "CD3-OFDM: a novel demodulation scheme for fixed and mobile receivers," IEEE Trans. Commun., vol. 44, pp. 1144-1151, Sept. 1996.

[12] H. H. H'mimy, "Channel estimation based on coded pilot for OFDM,” Proc. IEEE VTC'97, pp. 1375-1379, May 1997.

[13] J.-W. Choi and Y.-H. Lee, "Design of the optimum pilot pattern for channel estimation in OFDM systems," IEEE Globecom'04, pp. 3661-3665, Dec. 2004.

[14] J.-W. Choi and Y.-H. Lee, "Design of 2-D channel estimation filters for OFDM systems," IEEE Intl. Commun. (ICC'05), pp. 2568-2572, May 2005.

[15] M.-H. Hsieh and C.-H. Wei, "Channel estimation for OFDM systems based on comb-type pilot arrangement in frequency selective fading channels," IEEE Trans. Consumer Electronics, pp. 217-225, Feb. 1998.

[16] G. Auer and E. Karipidis, "Pilot aided channel estimation for OFDM: a separated approach for smoothing and interpolation," IEEE Intl. Commun. (ICC'05), pp. , May 2005.

[17] L. Xiao, X. Dong and A. C. K. Soong, "On the design of sinc interpolator for pilot symbol assisted modulation systems," IEEE Trans. Wireless Commun., to appear. [Online] http://www.ece.uvic.ca/ xdong/interpolator.pdf

[18] G. Deslauriers and S. Dubuc, "Symmetric iterative interpolation processes," Constructive Approximation, vol. 5, pp. 49-68, 1989.

[19] S. Dubuc, "Interpolation through an iterative scheme," J. Math. Anal. and Appl., vol. 114, pp. 185-204, 1986.

[20] D. L. Donoho and T. P. Y. Yu, "Deslauriers-Dubuc: Ten years after," CRM Proc. and Lecture Notes, vol. 18, 1999. [Online] http://www-stat.stanford.edu/ donoho/Reports/1996/montreal.ps

[21] S. D. Stearns and R. A. David, Signal Processing Algorithms in MATLAB, Prentice-Hall Inc., 1996.

[22] W.-S. Lu and A. Antoniou, Two-Dimensional Digital Filters, Marcel Dekker Inc., 1992.

[23] S. Mallat, A Wavelet Tour of Signal Processing, 2nd ed., Academic Press, 1999.

[24] Recommendations ITU-R M.1225, Guidelines for Evaluation of Radio Transmission Technologies for IMT-2000, 1997. 
[25] 3GPP TR 25.943 v6.0.0, 3rd Generation Partership Project; Technical Specification Group Radio Access Networks; Deployment aspects, 2004.

\section{APPENDIX}

MSE ANALYSIS OF 2-D LINEAR INTERPOLATOR

Following (11a), a 2-D linear interpolator is given by

$$
\begin{aligned}
\hat{H}[n, k] & =\sum_{u=U_{1}}^{U_{2}} \sum_{v=V_{1}}^{V_{2}} w[u, v] \hat{H}_{p}[n-u, k-v], \\
\hat{H}_{p}[n, k] & = \begin{cases}\tilde{H}_{p}[p, q], & n=p d_{t}, k=q d_{f} \\
0, & \text { otherwise }\end{cases}
\end{aligned}
$$

The mean-squared error of this interpolator can be written as

$$
\begin{aligned}
M S E & =\mathbb{E}\left\{|H[n, k]-\hat{H}[n, k]|^{2}\right\} \\
& =\mathbb{E}\left\{|H[n, k]|^{2}\right\}-2 \Re\left(\mathbb{E}\left\{H^{*}[n, k] \hat{H}[n, k]\right\}\right)+\mathbb{E}\left\{|\hat{H}[n, k]|^{2}\right\} \\
& =1-2 \Re\left(\mathbb{E}\left\{H^{*}[n, k] \hat{H}[n, k]\right\}\right)+\mathbb{E}\left\{|\hat{H}[n, k]|^{2}\right\}
\end{aligned}
$$

To evaluate the second term in (37), we use (35) to write

$$
\mathbb{E}\left\{H^{*}[n, k] \hat{H}[n, k]\right\}=\sum_{u} \sum_{v} w[u, v] \mathbb{E}\left\{H^{*}[n, k] \hat{H}_{p}[n-u, k-v]\right\}
$$

where, according to (36), $\hat{H}_{p}[n-u, k-v]$ assumes nonzero values $\tilde{H}_{p}[p, q]$ only when $n-u$ is an integer multiple of $d_{t}$ and $k-v$ is an integer multiple of $d_{f}$. Hence we can write

$$
\begin{aligned}
\mathbb{E}_{n, k}\left\{H^{*}[n, k] \hat{H}_{p}[n-u, k-v]\right\} & =\mathbb{E}_{n, k}\left\{H^{*}\left[u+p d_{t}, v+q d_{f}\right] \hat{H}_{p}\left[p d_{t}, q d_{f}\right]\right\} \\
& =\rho \mathbb{E}_{p, q}\left\{H^{*}\left[u+p d_{t}, v+q d_{f}\right] \tilde{H}_{p}[p, q]\right\} \\
& =\rho \mathbb{E}_{p, q}\left\{H^{*}\left[u+p d_{t}, v+q d_{f}\right] H\left[p d_{t}, q d_{f}\right]\right\} \\
& =\rho r_{t}(-u) r_{f}(-v)
\end{aligned}
$$

where $\rho=\frac{1}{d_{t} d_{f}}$ denotes the ratio of the number of pilot symbols over the number of total pilot and data symbols. 
By (35) and an arguement similar to that given above, the third term in (37) can be evaluated as

$$
\begin{aligned}
& \mathbb{E}_{n, k}\left\{|\hat{H}[n, k]|^{2}\right\}=\mathbb{E}_{n, k}\left\{\left|\sum_{u} \sum_{v} w[u, v] \hat{H}_{p}[n-u, k-v]\right|^{2}\right\} \\
& =\sum_{u} \sum_{v} \sum_{u^{\prime}} \sum_{v^{\prime}} w[u, v] w\left[u^{\prime}, v^{\prime}\right] \mathbb{E}_{n, k}\left\{\hat{H}_{p}\left[p d_{t}+u^{\prime}-u, q d_{f}+v^{\prime}-v\right] \hat{H}_{p}^{*}\left[p d_{t}, q d_{f}\right]\right\} \\
& =\rho^{2} \sum_{u} \sum_{v} \sum_{u^{\prime}} \sum_{v^{\prime}} w[u, v] w\left[u^{\prime}, v^{\prime}\right] \mathbb{E}_{p, q}\left\{H\left[p d_{t}+u^{\prime}-u, q d_{f}+v^{\prime}-v\right] H^{*}\left[p d_{t}, q d_{f}\right]\right\} \\
& \quad+\rho^{2} \sum_{u} \sum_{v} w^{2}[u, v] \sigma_{n}^{2}
\end{aligned}
$$

where $u^{\prime}-u=p^{\prime} d_{t}$ and $v^{\prime}-v=q^{\prime} d_{f}, p^{\prime} \in \mathcal{Z}, q^{\prime} \in \mathcal{Z}$, and $\sigma_{n}^{2}$ is the variance of the noise in the channel estimation at pilot positions assuming all pilot symbols have the same magnitude. Further simplification leads to

$$
\begin{aligned}
\mathbb{E}_{n, k}\left\{|\hat{H}[n, k]|^{2}\right\} & =\rho^{2} \sum_{p^{\prime}} \sum_{q^{\prime}} \sum_{u} \sum_{v} w[u, v] w\left[u+p^{\prime} d_{t}, v+q^{\prime} d_{f}\right] r_{t}\left(p^{\prime} d_{t}\right) r_{f}\left(q^{\prime} d_{f}\right) \\
& +\rho^{2} \sigma_{n}^{2} \sum_{u} \sum_{v} w^{2}[u, v]
\end{aligned}
$$

Therefore, the mean-squared error of 2-D linear interpolator is given by

$$
\begin{aligned}
M S E & =1-\frac{2}{d_{t} d_{f}} \Re\left(\sum_{u=U_{1}}^{U_{2}} \sum_{v=V_{1}}^{V_{2}} w[u, v] r_{t}(-u) r_{f}(-v)\right) \\
& +\frac{1}{d_{t}^{2} d_{f}^{2}} \sum_{p^{\prime}=U_{1} / d_{t}}^{U_{2} / d_{t}} \sum_{q^{\prime}=V_{1} / d_{f}}^{V_{2} / d_{f}} \sum_{u=U_{1}}^{U_{2}} \sum_{v=V_{1}}^{V_{2}} w[u, v] w\left[u+p^{\prime} d_{t}, v+q^{\prime} d_{f}\right] r_{t}\left(p^{\prime} d_{t}\right) r_{f}\left(q^{\prime} d_{f}\right) \\
& +\frac{\sigma_{n}^{2}}{d_{t}^{2} d_{f}^{2}} \sum_{u=U_{1}}^{U_{2}} \sum_{v=V_{1}}^{V_{2}} w^{2}[u, v]
\end{aligned}
$$


TABLE I

PILOT ASSISTED OFDM SYSTEM SIMULATION SETUP

\begin{tabular}{||c|c||}
\hline Parameters & Values \\
\hline OFDM symbol duration $(T)$ & $51.2 \mu \mathrm{s}+1.28 \mu \mathrm{s}$ (guard interval) \\
\hline FFT size $(K)$ & 256 \\
\hline Total bandwidth & $5 \mathrm{MHz}$ \\
\hline Sub-carrier spacing $(\Delta f)$ & $19.5313 \mathrm{KHz}$ \\
\hline Pilot percentage $\left(\frac{1}{d_{t} d_{f}}\right)$ & $6.25 \%\left(d_{t}=d_{f}=4\right)$ and $1.56 \%\left(d_{t}=d_{f}=8\right)$ \\
\hline Carrier frequency & $2 \mathrm{GHz}$ \\
\hline Maximum Doppler frequency $\left(f_{D}\right)$ & $222.22 \mathrm{~Hz}(120 \mathrm{~km} / \mathrm{h})$ and $55.56 \mathrm{~Hz}(30 \mathrm{~km} / \mathrm{h})$ \\
\hline Modulation & 64-QAM \\
\hline Channel fading & Rayleigh with 2-D isotropic scattering \\
\hline Channel model type & ITU Vehicular A and COST 259 Typical Urban \\
\hline
\end{tabular}

\section{TABLE II}

COMPLEXITY COMPARISON OF DIFFERENT INTERPOLATORS

\begin{tabular}{||c|c||}
\hline Interpolation method & Number of complex multiplication per symbol \\
\hline Sinc interpolator & $2 M_{t}+\frac{4 M_{t} M_{f}}{d_{t}}$ or $2 M_{f}+\frac{4 M_{t} M_{f}}{d_{f}}$ \\
\cline { 2 - 3 } with Kaiser window & $2 M_{t}+\frac{2 M_{f}}{d_{t}}$ or $2 M_{f}+\frac{2 M_{t}}{d_{f}}$ (block processing) \\
\hline DD interpolation & $2 D_{t}+\frac{4 D_{t} D_{f}}{d_{t}}$ or $2 D_{f}+\frac{4 D_{t} D_{f}}{d_{f}}$ \\
\cline { 2 - 2 } & $2 D_{t}+\frac{2 D_{f}}{d_{t}}$ or $2 D_{f}+\frac{2 D_{t}}{d_{f}}$ (block processing) \\
\hline 2-D DFT & $2 \log _{2}\left(N_{p} K_{q}\right)($ FFT and IFFT) \\
\hline MMSE & $\mathcal{O}\left(\left(M_{s}^{t}\right)^{3} / 6\right)+\mathcal{O}\left(\left(M_{s}^{f}\right)^{3} / 6\right)+\left(M_{s}^{t}\right)^{2}+\left(M_{s}^{f}\right)^{2}+M_{s}^{t}+M_{s}^{f}$ \\
\hline
\end{tabular}



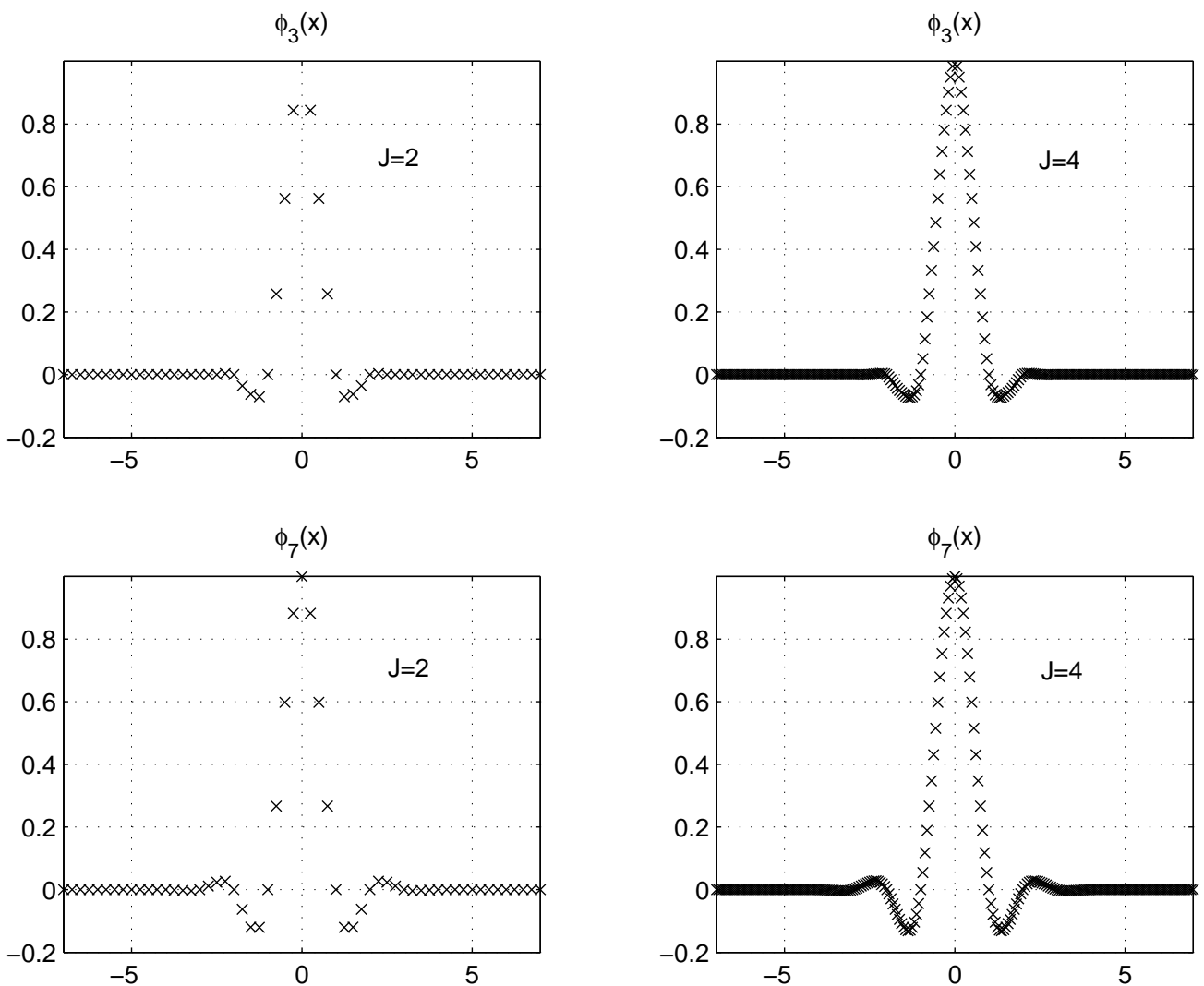

Fig. 1. The fundamental functions of DD interpolation. 


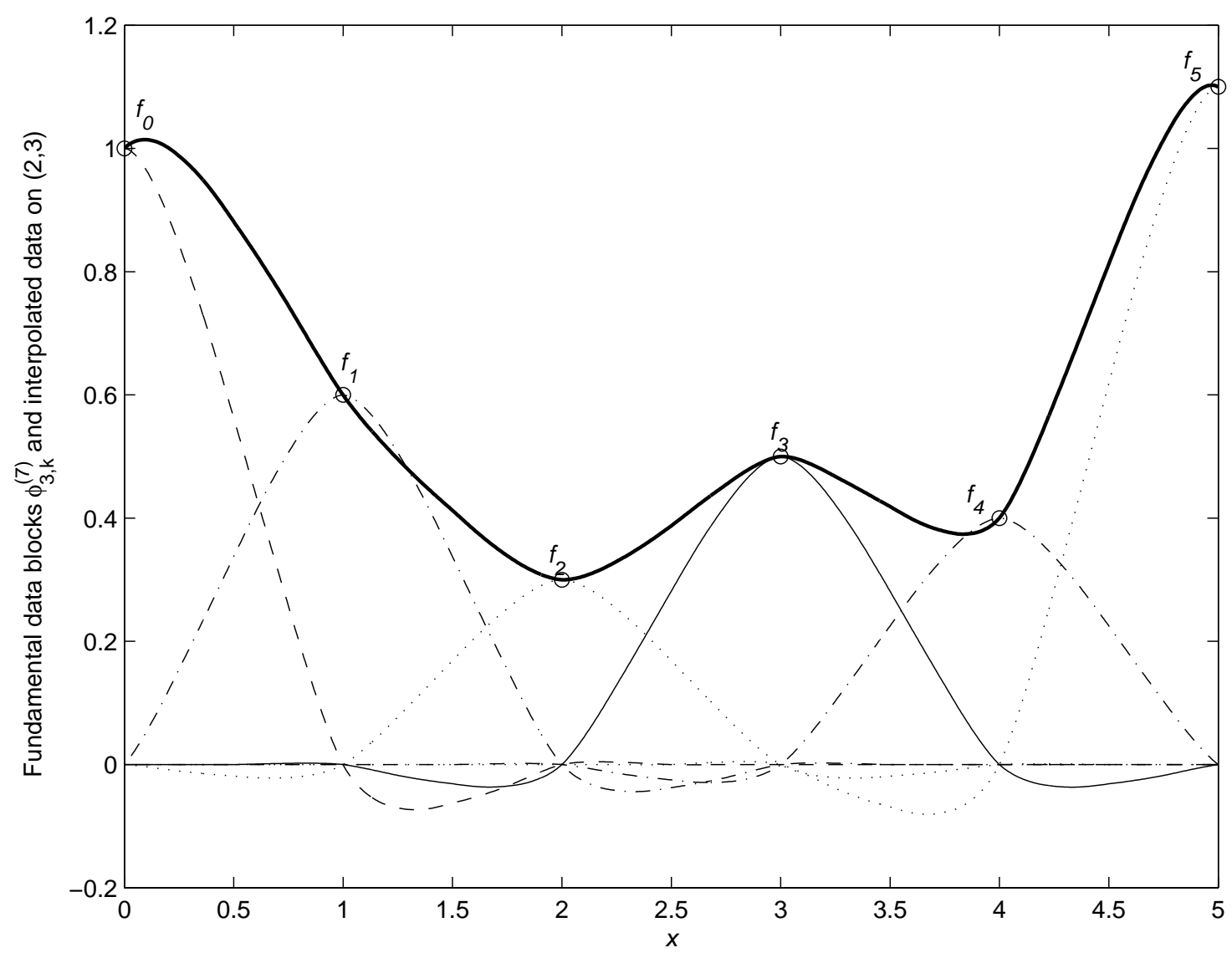

Fig. 2. An illustrative example of the DD interpolation with $D=3$ and $J=7$. 


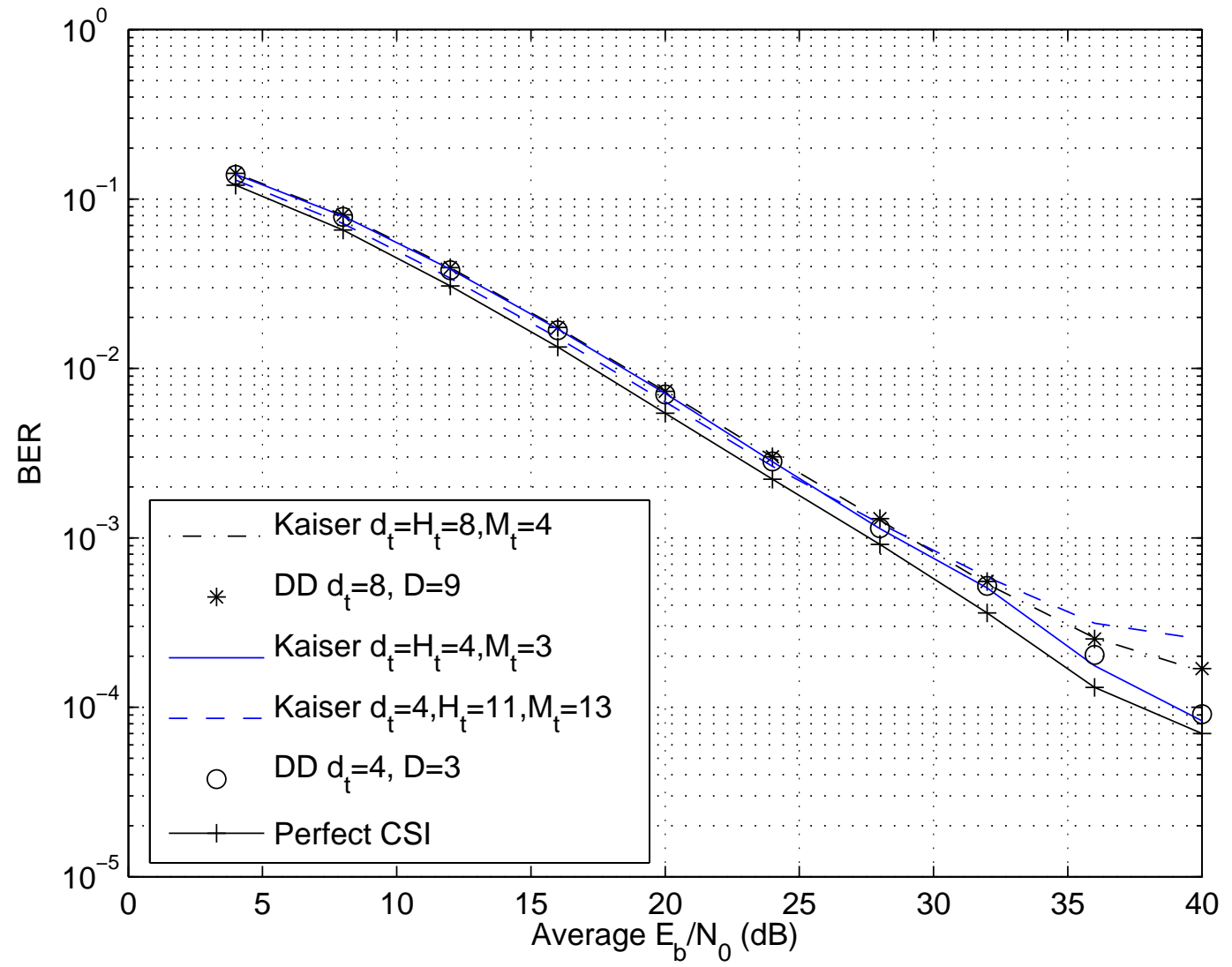

Fig. 3. Performance of PSAM 64-QAM in frequency flat Rayleigh fading with $f_{D} T=0.03$. 


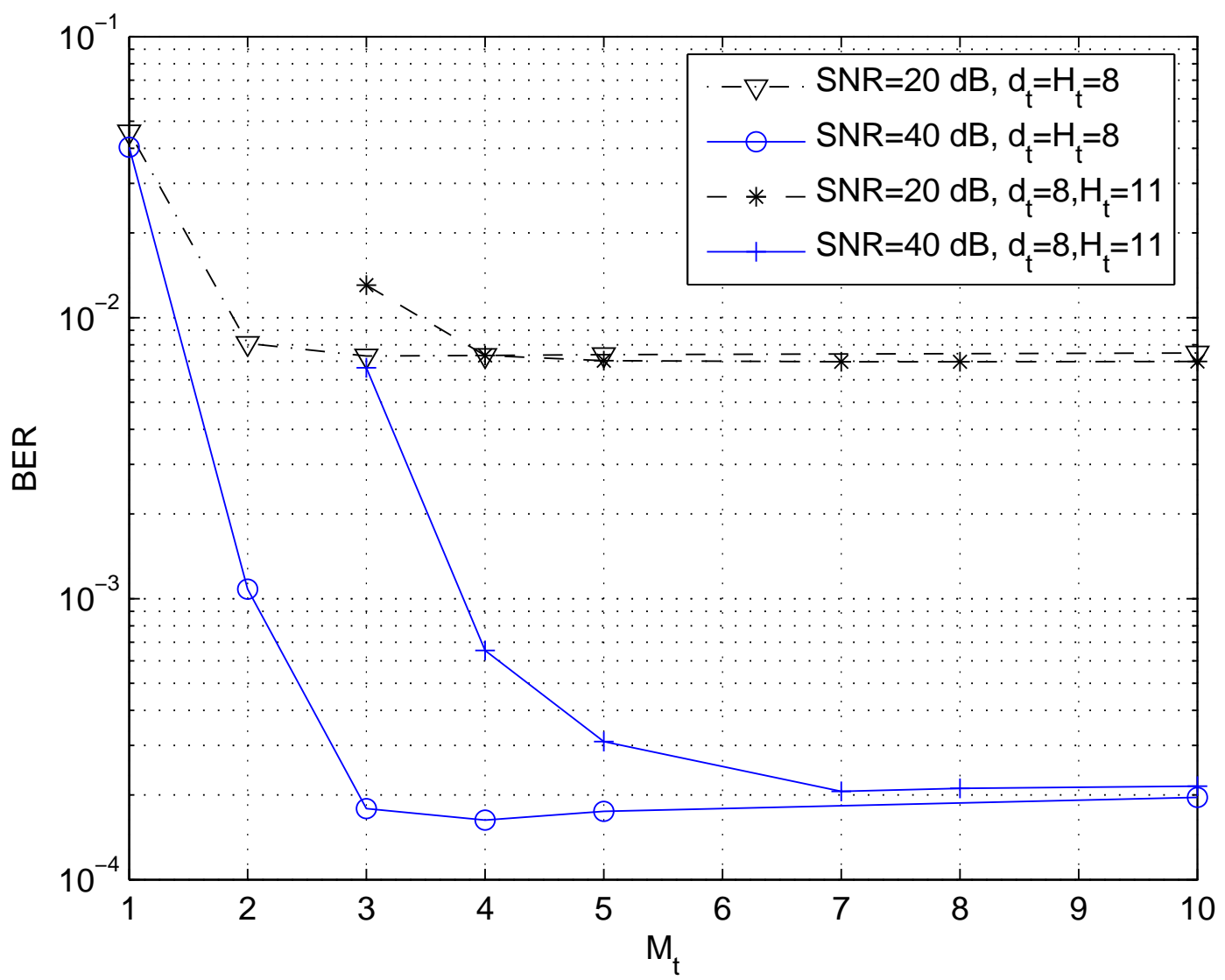

Fig. 4. Performance of PSAM 64-QAM in frequency flat Rayleigh fading with $f_{D} T=0.03$, using sinc interpolator with Kaiser window. SNR is the average $E_{b} / N_{0}$. 


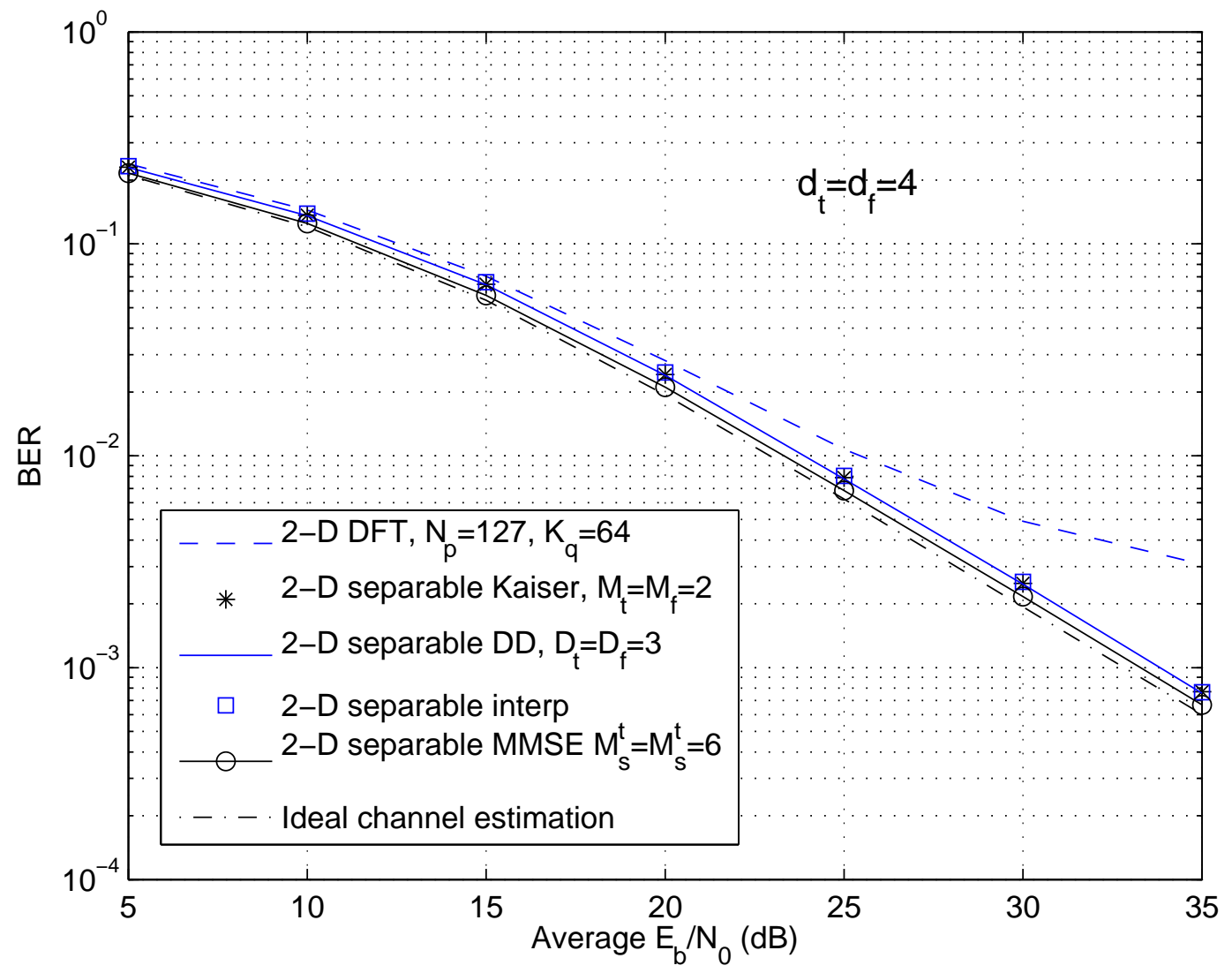

Fig. 5. Performance of 64-QAM OFDM using different interpolation methods in ITU Vehicular A channels, with $d_{t}=d_{f}=4$ and $v=120 \mathrm{~km} / \mathrm{h}$. 


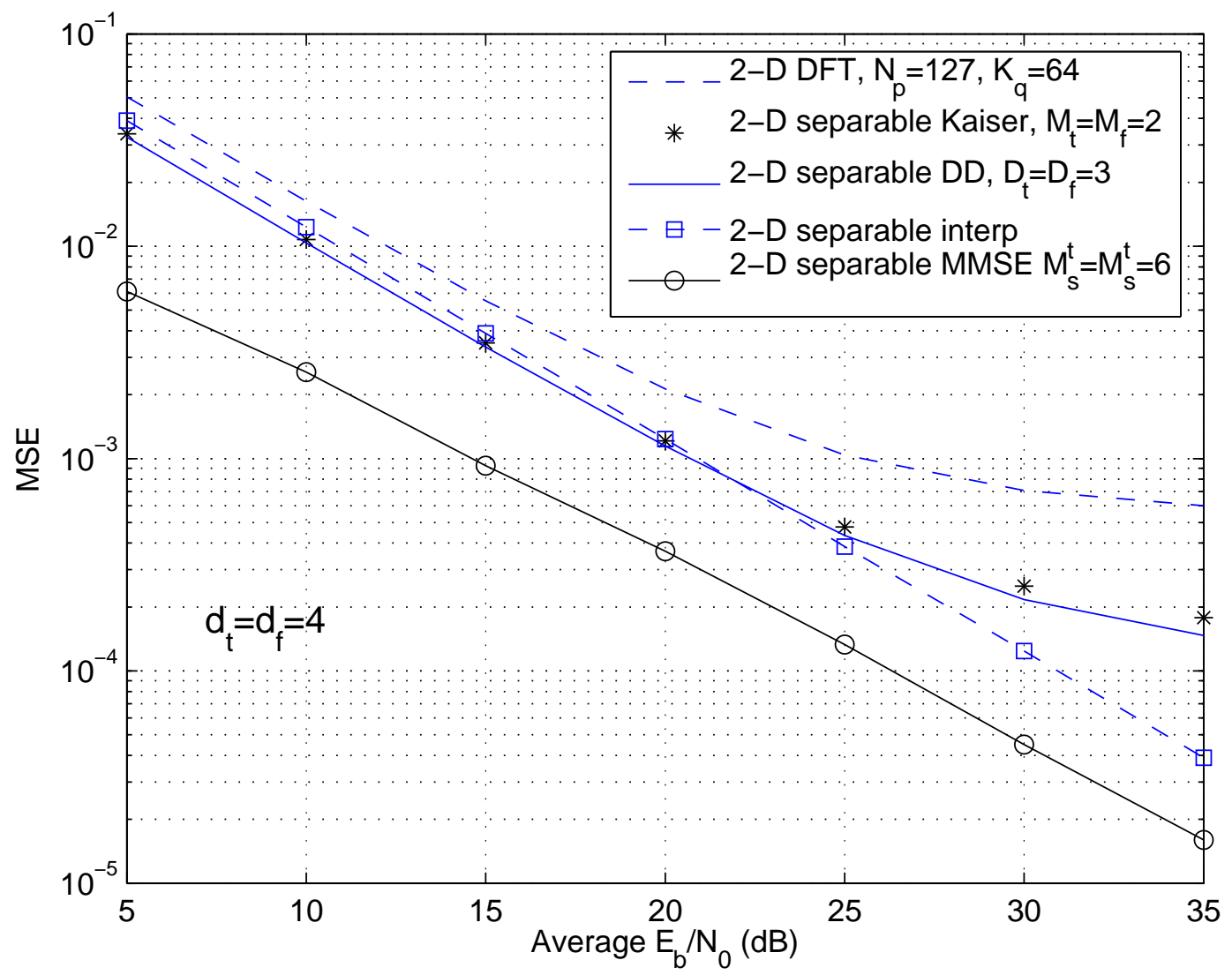

Fig. 6. MSE of 64-QAM OFDM using different interpolation methods in ITU Vehicular A channels, with $d_{t}=d_{f}=4$ and $v=120 \mathrm{~km} / \mathrm{h}$. 


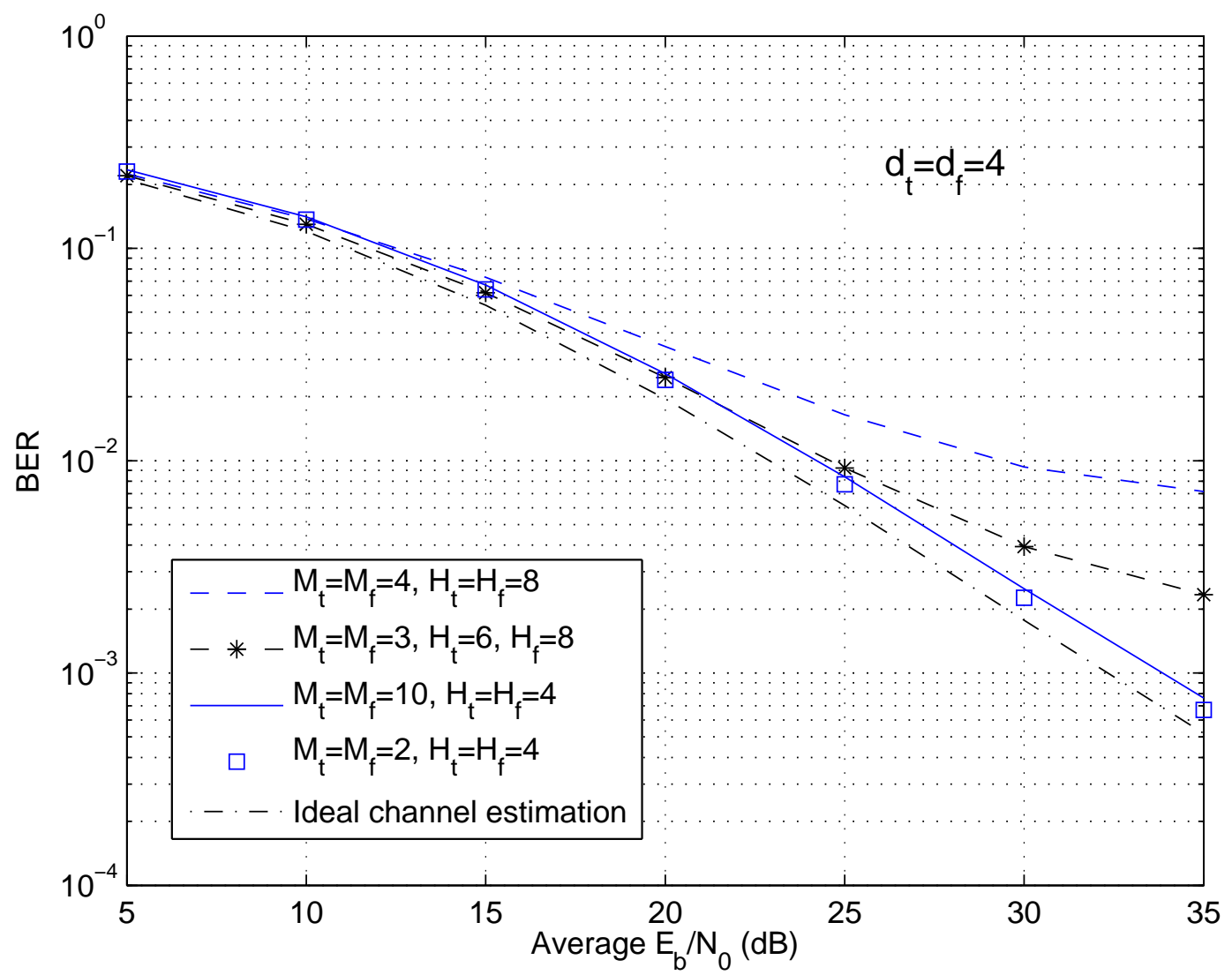

Fig. 7. Performance of 64-QAM OFDM using sinc interpolator with Kaiser window in ITU Vehicular A channels, with $d_{t}=d_{f}=4$ and $v=30 \mathrm{~km} / \mathrm{h}$. 


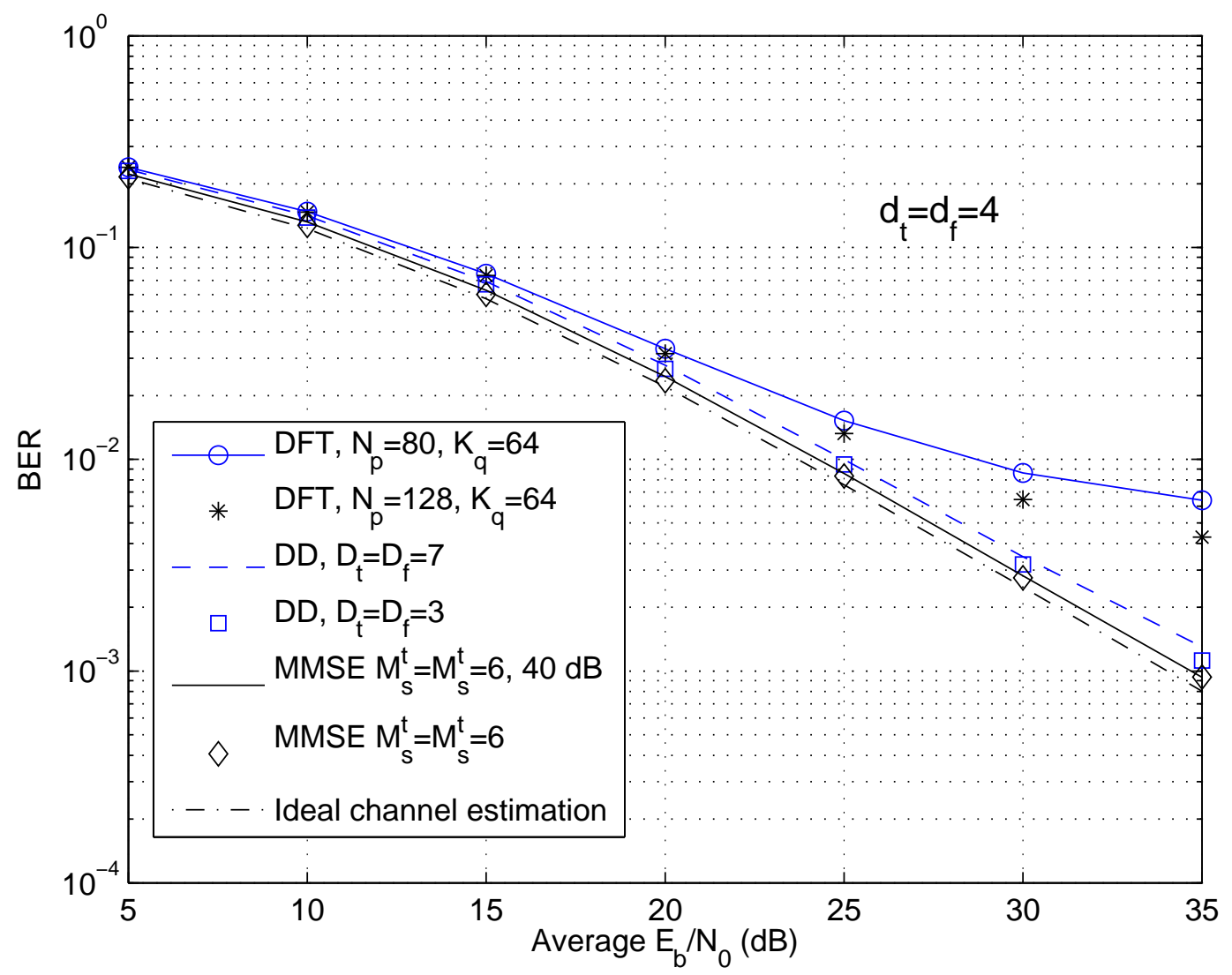

Fig. 8. Performance of 64-QAM OFDM using different interpolation methods in COST 259 typical urban channels, with $d_{t}=d_{f}=4$ and $v=120 \mathrm{~km} / \mathrm{h}$. 


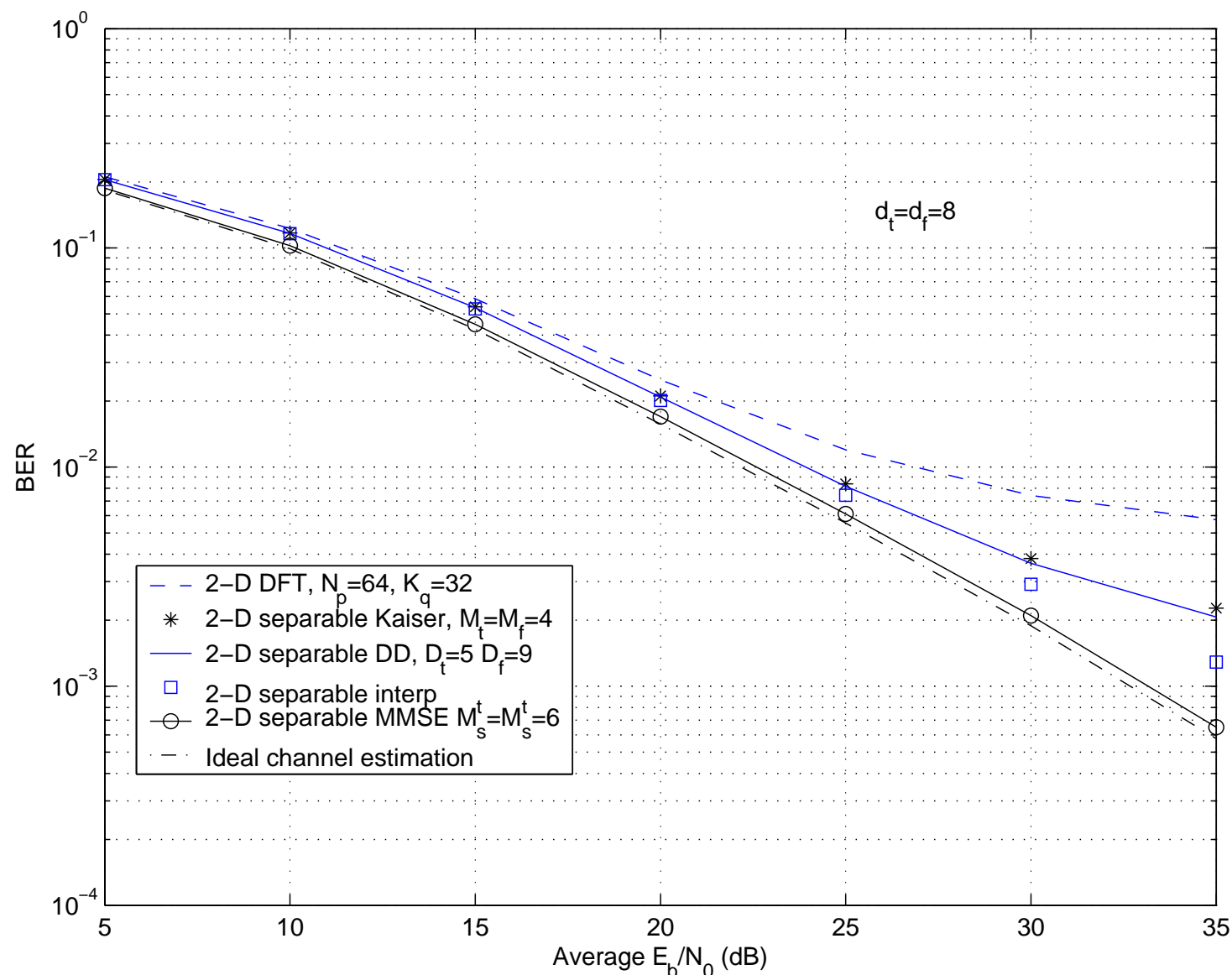

Fig. 9. Performance of 64-QAM OFDM using different interpolation methods in COST 259 typical urban channels, with $d_{t}=d_{f}=8$ and $v=30 \mathrm{~km} / \mathrm{h}$. 


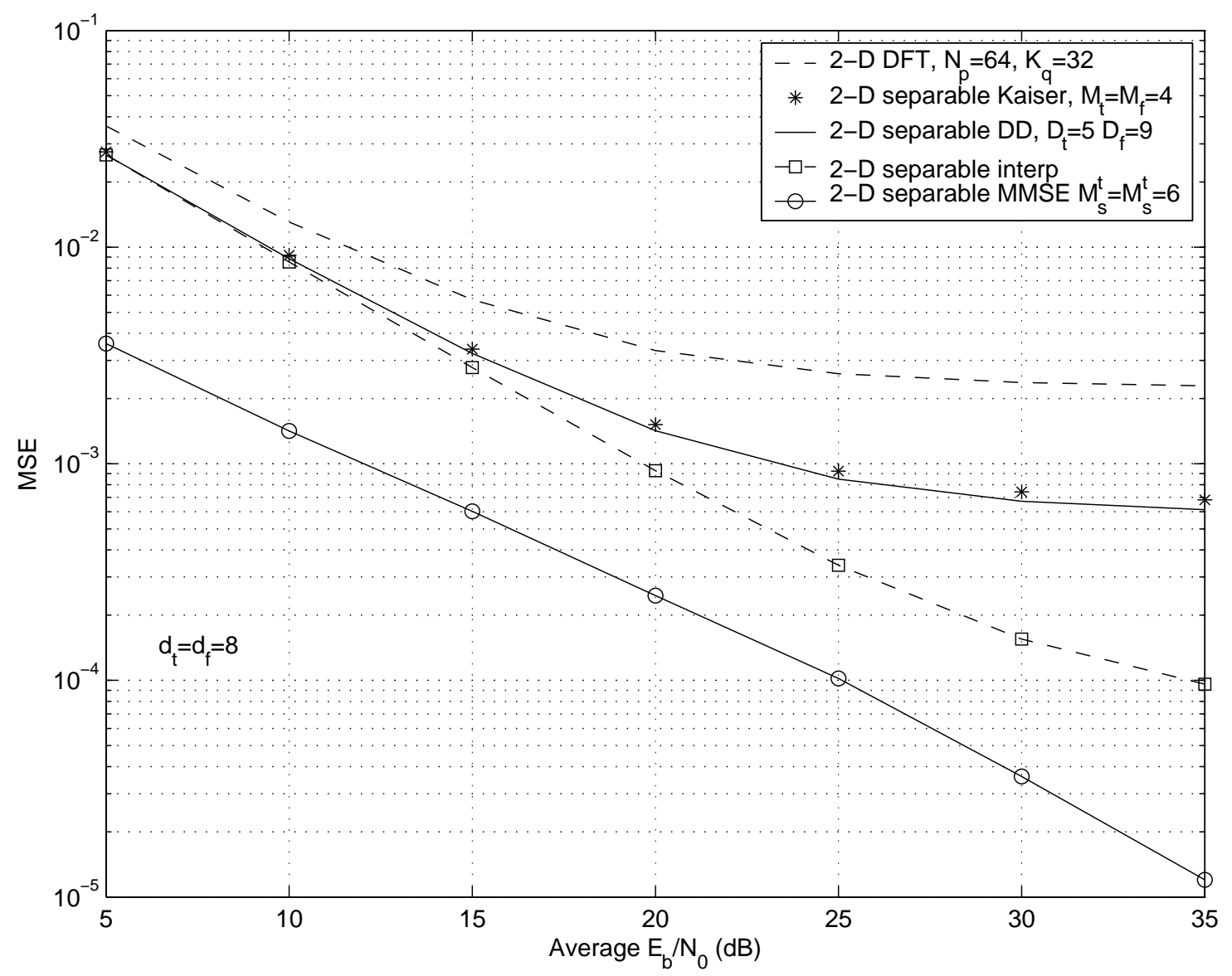

Fig. 10. MSE of 64-QAM OFDM using different interpolation methods in COST 259 typical urban channels, with $d_{t}=d_{f}=8$ and $v=30 \mathrm{~km} / \mathrm{h}$. 